\title{
Calculation of the Wavelength Filter Properties of the Fiber-Slab Waveguide Structure Using Vector Mode Expansion
}

\author{
Mehmet S. Dinleyici and David B. Patterson
}

\begin{abstract}
A vectorial solution technique is applied to investigate the dispersion characteristics of the ridge modes of a waveguide structure comprising a slab and optical fiber. The power transmission characteristics of the device with respect to wavelength are calculated under various device parameters, such as slab index and fiber-slab separation. We discuss the effects of such parameters on the bandwidth and rejection of the notch filter produced by this structure.
\end{abstract}

Index Terms-Optical coupling, optical fiber couplers, optical fiber devices, optical fiber filter, wavelength division multiplexing.

\section{INTRODUCTION}

$\mathbf{T}$ HE evanescent coupler comprising a single mode optical fiber and a multimode planar waveguide has been studied by a number of researchers for the wide variety of its possible applications in fiber optic communication systems. This device exhibits a resonant behavior as a function of the slab index or the input wavelength, and thus has applications in wavelength division multiplexing (WDM), optical switching and modulation. Although many experimental studies [1]-[4] have shown the potential of this device, there is no mathematical model that will fully explain the behavior of the device in a manner consistent with experimental observations. A mathematical model used by many researchers has been developed by Marcuse using coupled mode theory (CMT) [5]. This model assumes only weak perturbation of the modes which exist in the isolated waveguides, namely the slab and the fiber, and seeks a solution in the form of the linear combination of these modes. While this method has been shown to provide good agreement with experimental results under weakly coupled, low-perturbation conditions [2], the approximation breaks down under conditions of strong perturbation as exemplified in the same reference for cases of extremely close waveguide proximity and long wavelengths.

The CMT model, in its simplest, two-mode form, predicts a periodic exchange of power between waveguides under resonant conditions where the fiber mode propagation constant is nearly matched to a slab mode propagation constant. However, in practice such a periodic exchange of energy has not been

Manuscript received October 8, 1997; revised February 6, 1998 and July 7, 1998.

M. S. Dinleyici is with the Electronic and Electrical Engineering Department, Ǐzmir Institute of Technology, 35230-Çankaya Ǐzmir, Turkey.

D. B. Patterson is with the Electrical and Computer Engineering Department, Illinois Institute of Technology, Chicago, IL 60616 USA.

Publisher Item Identifier S 0733-8724(98)08306-6. observed (i.e., no over coupling of the power back into the fiber has been reported). One approach to this discrepancy has been to introduce a loss factor into the problem [5]. A more physical solution to the problem has been the distributed coupling approach [6], where coupled-mode equations are developed for all modes of the slab waveguide, and numerical solutions are found that require no return of power to the fiber mode. While this approach has accurately predicted wavelength-dependent behavior of weakly coupled devices, it cannot be used in many practical cases of close proximity, where the two guides may, in fact, be in contact.

The inadequacy of CMT in predicting the behavior of this device arises from two obvious inconsistencies in the model. First, the waveguide fields are assumed to have only slight perturbations from the isolated mode profiles and propagation. This is not a valid assumption for the typically high refractive index slab $\left(\mathrm{LiNbO}_{3}\right)$ configuration and close proximities used in practical device designs. Second, CMT requires two wellbounded modes, with no mechanism for power dissipation during propagation. Since CMT exploit the orthogonality of the modes it weakens with the absorption due to different attenuation constants of the modes [7]. However, the unbounded nature of the isolated slab mode leads to a one-way transfer of power from the fiber to the slab under resonant conditions. Power transferred from the fiber to the slab diffracts in the unbounded direction and thus can not be efficiently recoupled to the fiber.

In [8], we describe a vectorial method, introduced by Marcuse for the analysis of D-fiber [9], to investigate dispersion and power transmission characteristics of the device. We have identified the ridge modes of this device by regarding the whole waveguide structure as a single waveguide. The results obtained for different slab/core indexes and distances between two waveguides by this vectorial method are in very good agreement with experimental results [4], [6].

In this paper we apply this model to predict the bandreject characteristics of the fiber-slab waveguide device under various conditions such as slab refractive index and fiberslab separation. We discuss the effects of these parameters on the filter bandwidth, insertion loss, and band rejection and compare our results with published experimental data.

We also note that our ridge mode solution technique predicts no periodic exchange of energy between waveguides, and accurately models the observed behavior of such devices, providing a significant improvement over coupled-mode theory. 


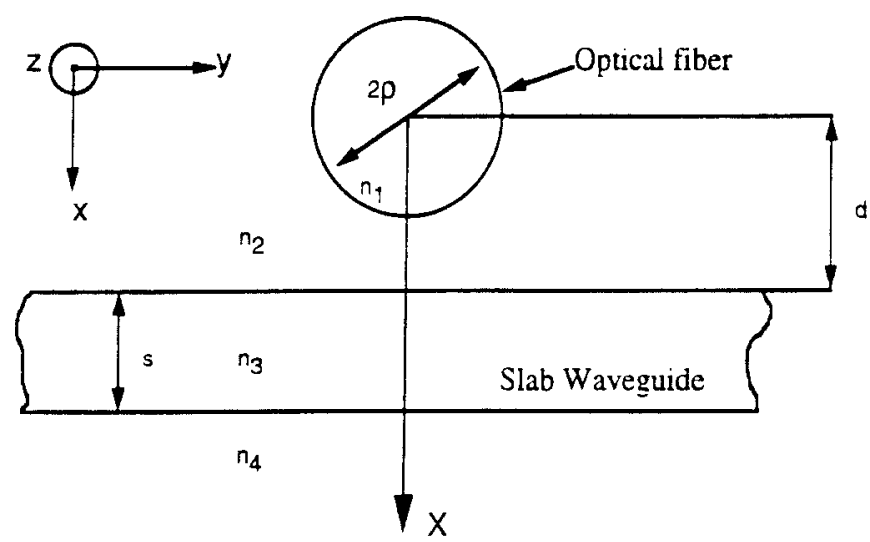

Fig. 1. Geometry of the evanescent coupler.

\section{Mathematical Model}

The mathematical model used in this study was first developed by Marcuse [9] to analyze the characteristics of D-fiber. In Marcuse's model the cladding has been modified by introducing a plane boundary to an infinite half-space rather than another waveguide. The interaction of the cladding fields with the fields in the half-space are described by means of reflection coefficients [10]. Those coefficients match the cladding fields in cylindrical form with the fields of the half-space in Cartesian coordinates. By exploiting this technique and replacing the infinite half-space with a planar slab waveguide, we have developed a mathematical model for the evanescent fiber/slab coupler and have analyzed the behavior of various device geometries in [8]. The relevant geometries and refractive indexes are shown in Fig. 1. In the present work we expand this analysis to model the wavelength characteristics of the fiber/slab evanescent coupler. Following the steps described in [8] we outline the mathematical model below.

The fields in the various media are assumed to have $\exp (j \omega t)$ time dependence and $\exp (-j \omega z) z$-dependence. Throughout this derivation only transverse magnetic (TM) mode fields will be considered; however, transverse electric (TE) mode fields can be obtained (as stated in [8]) by interchanging the $\cos (n \phi)$ and $\sin (n \phi)$ terms in the following expressions for $z$-components of the fields in the core and cladding (The solutions of [8] exhibit nearly TM and TE behavior, and are referred to as quasi-TM and quasi-TE modes; here we shall refer to these modes as simply TM and TE modes.). In the fiber core the fields are

$$
\begin{aligned}
& e_{z 1}=\sum_{n=0}^{N} A_{n}^{(1)} J_{n}(U R) \cos (n \phi) \\
& h_{z 1}=\sum_{n=0}^{N} A_{n}^{(2)} J_{n}(U R) \sin (n \phi)
\end{aligned}
$$

where $U=\rho\left(k^{2} n_{1}^{2}-\beta^{2}\right)^{1 / 2} ; R=r / \rho ; k=2 \pi / \lambda$, and $R$ is the normalized radius. Also, $A_{n}^{(1)}, A_{n}^{(2)}$ are the fields coefficients in the core. The expansion functions $J_{n}(U R)$ are Bessel functions of the first kind with order $n$. The cladding fields may be expanded in terms of modified Bessel functions

$$
\begin{aligned}
& I_{n} \text { and } K_{n} \\
& e_{z 2}=\sum_{n=0}^{N}\left(B_{n}^{(1)} K_{n}(W R)+B_{n}^{(2)} I_{n}(W R)\right) \cos (n \phi) \\
& h_{z 2}=\sum_{n=0}^{N}\left(B_{n}^{(3)} K_{n}(W R)+B_{n}^{(4)} I_{n}(W R)\right) \sin (n \phi)
\end{aligned}
$$

where $W=\rho\left(\beta^{2}-k^{2} n_{2}^{2}\right)^{1 / 2}$. The fields in the slab may be expressed as a superposition of plane waves having the lack of a favorable propagation direction in the $y$-z plane, but bounded in the $x$-direction

$$
\begin{aligned}
& e_{z 3}=\int_{-\infty}^{\infty} \exp (j \nu Y)\left(C_{\nu}^{(1)} e^{-j \tau(X-D)}+C_{\nu}^{(2)} e^{j \tau(X-D)}\right) d \nu \\
& h_{z 3}=\int_{-\infty}^{\infty} \exp (j \nu Y)\left(C_{\nu}^{(3)} e^{-j \tau(X-D)}+C_{\nu}^{(4)} e^{j \tau(X-D)}\right) d \nu
\end{aligned}
$$

where $\tau^{2}+\nu^{2}=\rho^{2}\left(k^{2} n_{3}^{2}-\beta^{2}\right)$ and $D$ is the normalized separation distance, $D=d / \rho$. All distances represented via capital letters are normalized by core radius, $\rho$. The fields in the cover medium are

$$
\begin{aligned}
& e_{z 4}=\int_{-\infty}^{\infty} D_{\nu}^{(1)} \exp (-\gamma(X-L)) \exp (j \nu Y) d \nu \\
& h_{z 4}=\int_{-\infty}^{\infty} D_{\nu}^{(2)} \exp (-\gamma(X-L)) \exp (j \nu Y) d \nu
\end{aligned}
$$

where $\nu^{2}-\gamma^{2}=\left(k^{2} n_{4}^{2}-\beta^{2}\right) \rho^{2}$ and $L$ is the normalized distance from the origin to the slab/cover medium boundary. That is, $L=(d+s) / \rho$.

The expansion coefficients may be related by matching tangential field components at the various boundaries, as described in [6], leading to a set of $2 N$ homogeneous linear equations of $2 N$ mode coefficients. The solution of this set of equations provides the propagation constants and mode coefficients for the bound modes of the structure, which we identify as ridge modes of the waveguide [8].

\section{Spectral Properties of the Fiber-Slab Structure}

In [8], we used the preceding mathematical model to obtain the mode solutions for a geometry with $n_{1}=1.4646, n_{2}=$ $1.46, n_{4}=1.46, \lambda=0.85, \rho=2.5 \mu \mathrm{m}, d=3 \mu \mathrm{m}$, and $s=3 \mu \mathrm{m}$. Here, the slab index $n_{3}$ was varied, and the solution to the first two guided (ridge) modes were calculated. By referring these results the effective indexes of the modes varies between the fiber effective index (at which the power is well-bounded near the core region of the fiber) to the limit $n_{1}$, beyond this the mode is evanescent in the core. In the latter region, the ridge mode approaches an unbounded slab mode of the planar waveguide, and very little power is carried in the core region. Power transmission through this structure can be calculated by first decomposing the mode field of an unperturbed optical fiber into the ridge modes, propagating the field through the waveguide, and again decomposing this field into the normal modes of an unperturbed output fiber. The power transmission properties of the device, which reflect the degree of field confinement in the core region are 


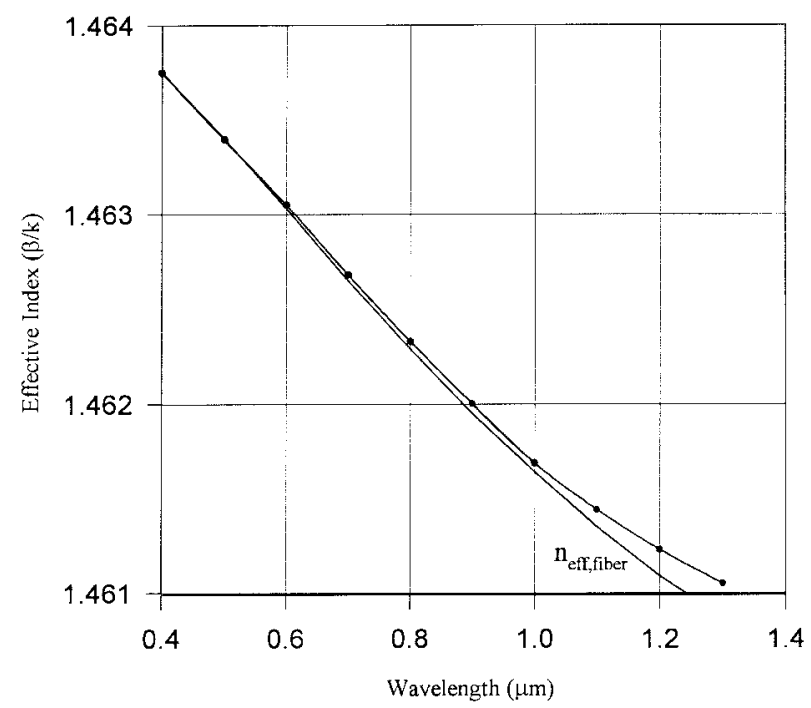

(a)

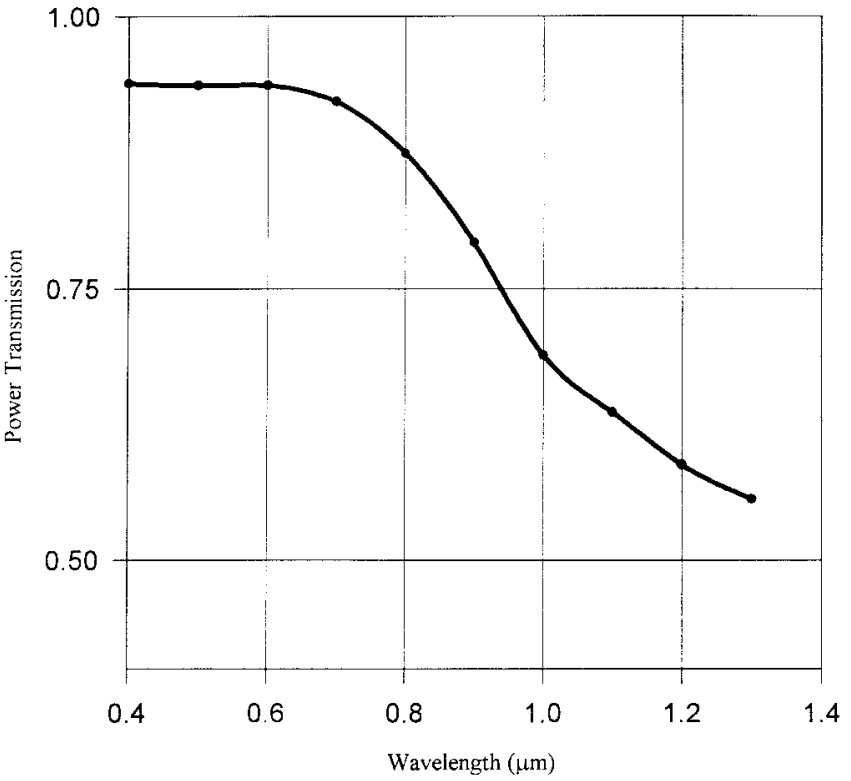

(b)

Fig. 2. (a) Effective index as a function of wavelength for $n_{1}=1.4646$ $n_{2}=n_{4}=1.46, \rho=2.5 \mu \mathrm{m}, s=3 \mu \mathrm{m}, d=3.6 \mu \mathrm{m}$, and $n_{3}=1.462$. (b) Power transmission for $n_{1}=1.4646, n_{2}=n_{4}=1.46$, $\rho=2.5 \mu \mathrm{m}, s=3 \mu \mathrm{m}, d=3.6 \mu \mathrm{m}$, and $n_{3}=1.462$.

also presented in [8]. The power transmission curve shows a minimum as the first ridge mode becomes unbounded, continuing until the second ridge mode begins propagating near $n_{3}=1.468$, and then it exhibits a maximum around $n_{3}=1.471$.

Here we shall consider the wavelength dependence of the dispersion at near the minimum and maximum power transmission regions. In Fig. 2(a) and (b), we plot the dispersion and power transmission curves, respectively, for $n_{3}=1.462$. We observe that the ridge mode deviates from the fiber mode solution only for very long wavelengths. The power curve reflects this behavior, with reduces transmission at longer wavelengths. Although the device is effectively a high-pass filter, the transmission coefficient never really approaches zero for this geometry.

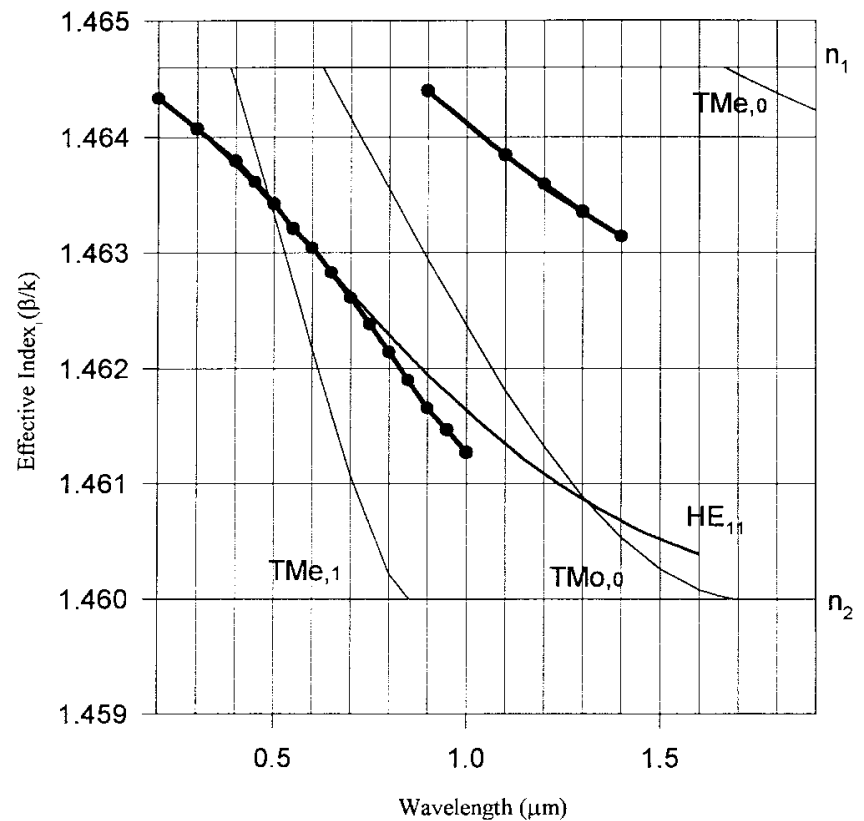

(a)

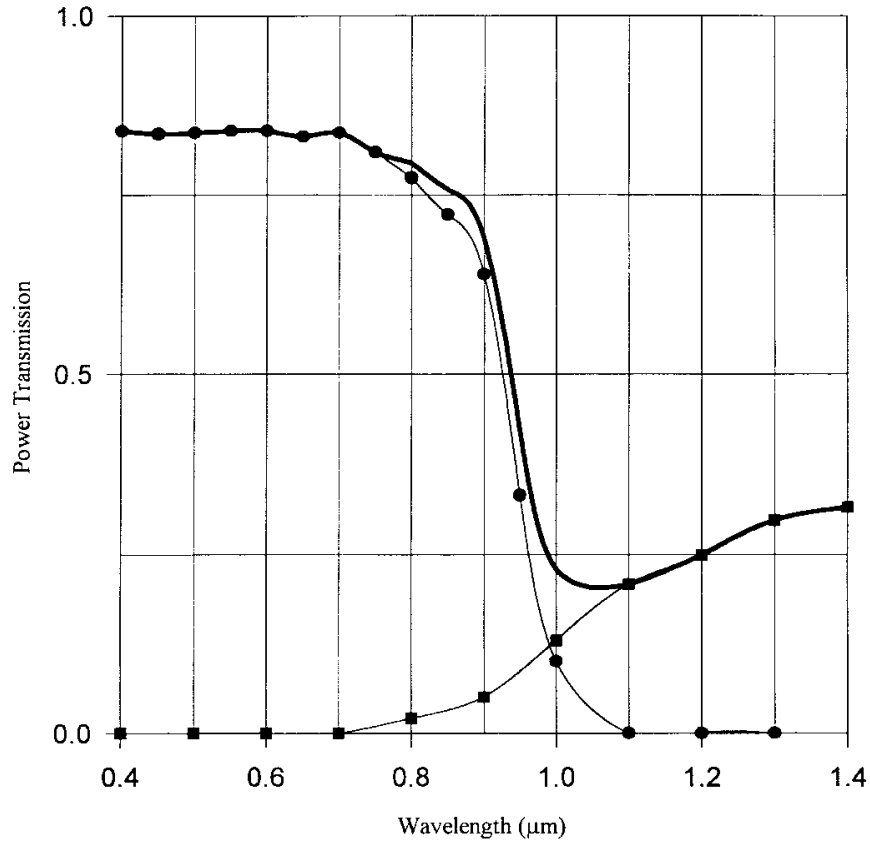

(b)

Fig. 3. (a) Effective index as a function of wavelength for $n_{1}=1.4646$, $n_{2}=n 4=1.46, \rho=2.5 \mu \mathrm{m}, s=3 \mu \mathrm{m}, d=3.0 \mu \mathrm{m}$, and $n_{3}=1.467$. (b) Power transmission for $n_{1}=1.4646, n_{2}=n_{4}=1.46$, $\rho=2.5 \mu \mathrm{m}, s=3 \mu \mathrm{m}, d=3.0 \mu \mathrm{m}$, and $n_{3}=1.467$.

In Fig. 3(a) and (b) we plot similar curves for the case $n_{3}=1.467$. We note that the first ridge mode solution diverges near $\lambda=1.0 \mu \mathrm{m}$, where the second ridge mode begins to propagate. Because the second ridge mode is only weakly bounded near cutoff, the total transmission does not immediately return to unity. In addition, in the region $\lambda<0.9 \mu \mathrm{m}$, where the first mode becomes unbounded, the presence of the second mode prohibits the transmitted power from reaching zero. Here, the spectral behavior is observed to resemble a band stop characteristic. This result is consistent with the 
experimental and theoretical results of [2], where CMT was used to model a similar configuration. Direct comparisons of he resonant wavelengths and power extinction measured in that reference may not be useful, as the later geometry was slightly different than that of our model, using the slowly varying waveguide separation produced in a coupler-half block geometry. However, our predicted wavelength dependence agrees well with the observed spectral characteristics.

Finally, we plot the dispersion and transmission behavior of the device versus wavelength for $n_{3}=1.471$ in Fig. 4(a) and (b). Here, we have solved the problem for two different separation parameters, $d=3 \mu \mathrm{m}$ and $d=3.6 \mu \mathrm{m}$. (The actual guide separations are 0.5 and $1.1 \mu \mathrm{m}$, respectively).

Fig. 4(a) illustrates two very important results of the analysis. First, we notice that the resonant point, at which the fiber/slab mode interaction is greatest, does not coincide with the isolated slab mode solutions ( as shown in the figure). This directly contradicts the coupled-mode theory assumption that coupling occurs when the slab mode and fiber mode effective indexes coincide. The second feature of Fig. 4(a) is the dependence of the resonant point on guide separation. The deviation of the fundamental mode from the isolated fiber mode solution is more rapid at larger separation. Thus, we expect a sharper bandpass characteristic for wider separation.

This behavior is clearly observed in Fig. 4(b), where the power transmission curves are plotted versus wavelength for the two guide separations. In both cases, the transmitted power is observed to approach zero near $\lambda=0.65 \mu \mathrm{m}$. However, the full-width at half-maximum (FWHM) is about $42 \%$ wider for the smaller guide separation.

Although the distributed CMT approach, like our approach, provides very good agreement with experimental results for configurations considered in this section, with a relatively low slab index, the former fails in cases of higher slab index where the perturbation to the normal guide modes is strong. Such a case is the commonly used geometry of thick $\mathrm{LiNbO}_{3}$ slab waveguides in close proximity to the fiber core. To our knowledge, there is no published theoretical approach that uses CMT to adequately analyze the high-index slab waveguide case; this problem is addressed in the following section.

\section{Characteristics of Lithium Niobate Slab Devices}

The previous results suggested that band-stop characteristics of the device become narrower as the slab refractive index increases. This prediction has been verified in several experimental studies which used lithium niobate $\left(\mathrm{LiNbO}_{3}\right)$, zinc selenide ( $\mathrm{ZnSe}$ ) and refractive index oils as planar waveguides in the coupler geometry [2], [11], [12]. Here we analyze both the symmetric slab $\left(n_{4}=1.46\right)$ and asymmetric slab $\left(n_{4}=\right.$ 1.0) for the $\mathrm{LiNbO}_{3}$ case. The dispersion curves of Fig. 5(a) illustrate the results for TM ridge modes. The resonant points, where the ridge modes deviate from the isolated fiber mode solution and power transmission is minimized, are seen to shift as the superstrate index is changed from 1.46 to 1.0. The observed shift of $100 \mathrm{~nm}$ suggests that the stop-band may be easily tuned by external means.

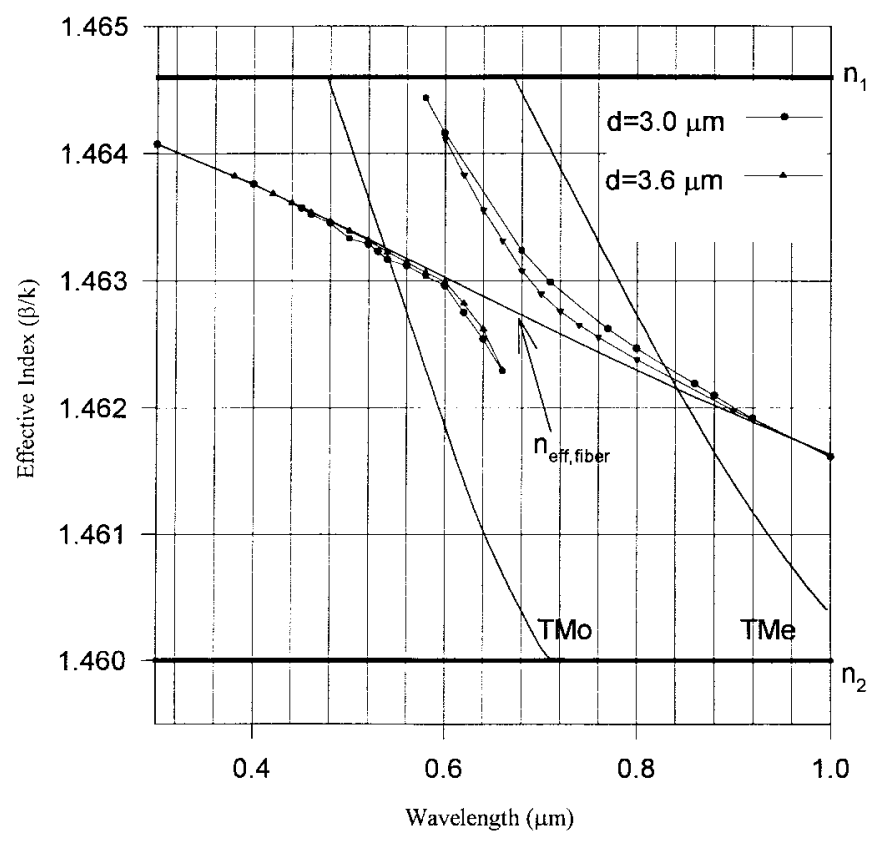

(a)

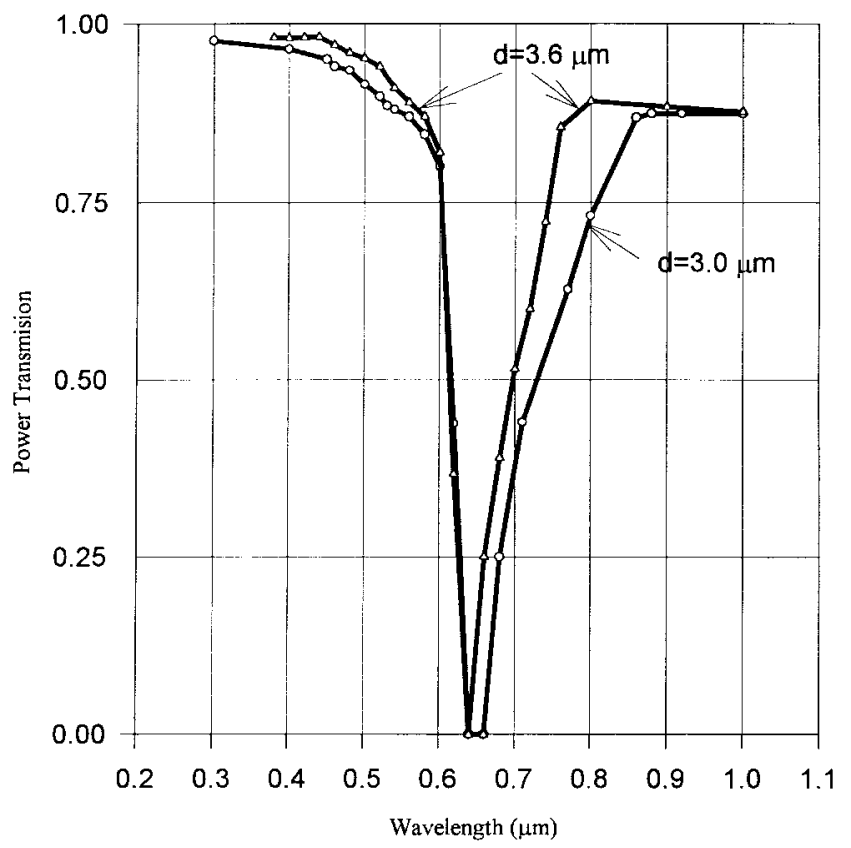

(b)

Fig. 4. (a) Effective index as a function of wavelength for $n_{1}=1.4646$, $n_{2}=n_{4}=1.46, \rho=2.5 \mu \mathrm{m}, s=3 \mu \mathrm{m}$, and $n_{3}=1.471$. (b) Power transmission for $n_{1}=1.4646, n_{2}=n_{4}=1.46$, $\rho=2.5 \mu \mathrm{m}, s=3 \mu \mathrm{m}$, and $n_{3}=1.471$.

It is interesting to note the mode cutoff behavior for the two geometries. It is well-known that the fundamental mode of an asymmetric waveguide may be cut off under conditions of sufficiently weak guidance. For the asymmetric slab $\left(n_{4}=1.0\right)$ this cutoff behavior is observed for $\lambda>1.2 \mu \mathrm{m}$, where the effective mode index drops below $n_{2}$. Beyond this wavelength no ridge mode propagates. As the slab becomes symmetric $\left(n_{4}=1.46\right)$, the cutoff condition moves to longer wavelengths, as expected. Fig. 5(b) shows the bandstop characteristics of the structure for the symmetric 


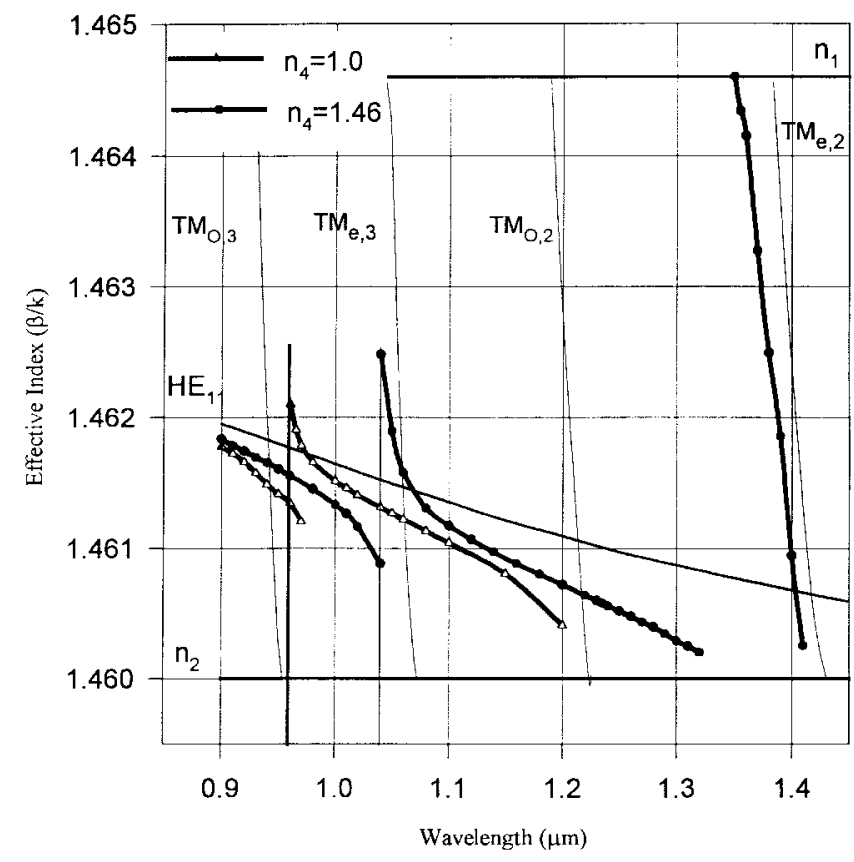

(a)

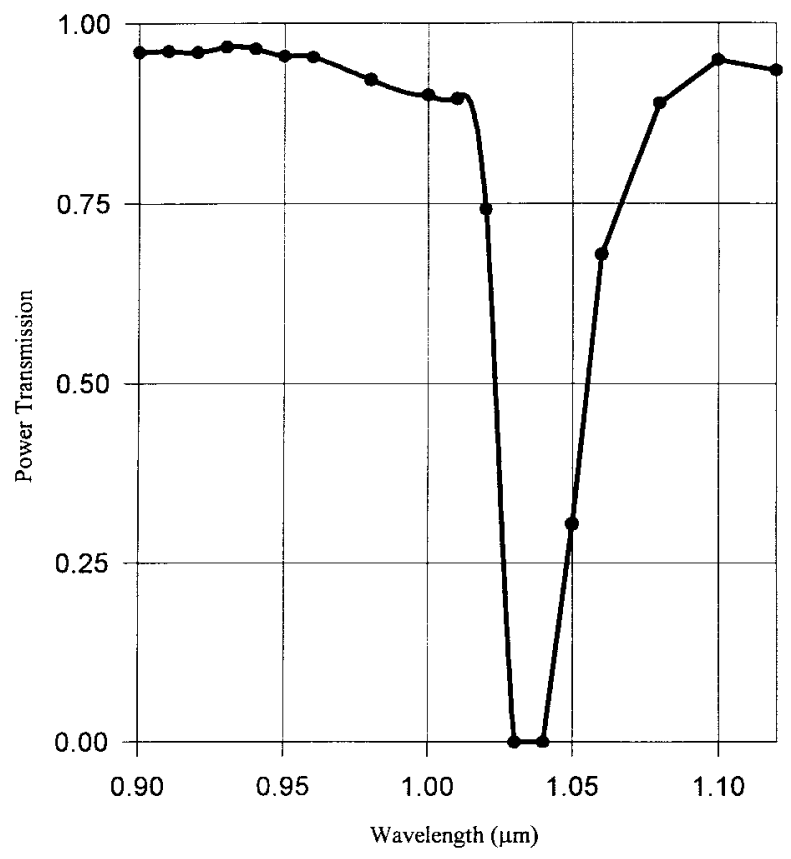

(b)

Fig. 5. (a) Effective index as a function of wavelength for $n_{1}=1.4646$, $n_{2}=1.46, \rho=2.5 \mu \mathrm{m}, s=3 \mu \mathrm{m}, d=3.6 \mu \mathrm{m}$, and $n_{3}=1.626$. (b) Power transmission for $n_{1}=1.4646, n_{2}=1.46$, $\rho=2.5 \mu \mathrm{m}, s=3 \mu \mathrm{m}, d=3.6 \mu \mathrm{m}$, and $n_{3}=1.626$.

case. Here, we predict the bandwidth of the filter to be 27.5 $\mathrm{nm}$ with an insertion loss in the transmitted band below $1 \mathrm{~dB}$. The level of extinction within the band cannot be accurately predicted by our model, as power coupled into unbounded slab modes is neglected in the transmission calculations; yet the leakage of this light to the output fiber will limit the level of band rejection. An obvious conclusion is that the rejection will improve with increased interaction length.

We next consider the polarization dependence of the device by calculating TE and TM modes under the assumption that

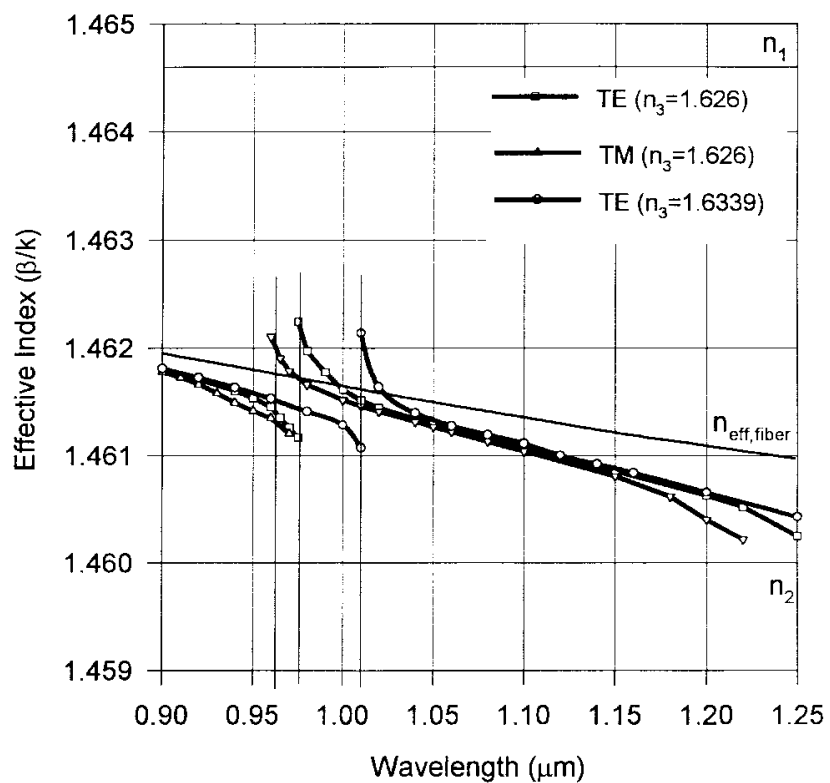

Fig. 6. Effective index as a function of wavelength for TE and TM modes of the $\mathrm{LiNbO}_{3}$.

$n_{3}=1.626$ for each case. The resulting dispersion curves, plotted in Fig. 6, show the waveguide-induced birefringence. The TE mode resonance [squares] is shifted from the TM mode resonance [triangles] by approximately $15 \mathrm{~nm}$ toward the longer wavelengths. In practical devices, this waveguide induced birefringence must be combined with the material birefringence of the lithium niobate slab. Typical values are $n_{3}=1.626$ for TM modes and $n_{3}=1.6339$ for TE modes [1]. The combined effect on the TE mode solution is shown in the third line [circles] in Fig. 6. Now the shift in resonance from TM to TE polarization increase to $50 \mathrm{~nm}$, in very good agreement with experimental reports [11].

This result is made clearer if we consider the exact parameters of the device reported in [11]. For $1.3 \mu \mathrm{m}$ single mode fiber we have used the following parameters $n_{1}=$ $1.4646, n_{2}=1.46$, and $\rho=2.5 \mu \mathrm{m}$. The other device parameters are $n_{3, \mathrm{TM}}=1.626, n_{4}=1.0, s=1.5 \mu \mathrm{m}$, and $d=3.6 \mu \mathrm{m}$. From a coupled-mode theory approach, one would predict a TM resonance at $\lambda=1.485 \mu \mathrm{m}$ using these parameters. However, the authors observed a TM resonance near $1.34 \mu \mathrm{m}$. We have applied our model to this system, and the resulting dispersion curves are plotted in Fig. 7. Here, we see very good agreement between our model and the experimental results. Also, it is important to observe that the resonant wavelength has been shifted by approximately 400 $\mathrm{nm}$ by changing the slab thickness from 3.0 to $1.5 \mu \mathrm{m}$.

\section{DISCUSSION}

The power transmission curves such as Fig. 4(b) show asymmetric behavior about the resonant point. The throughput power drops more rapidly at the short wavelength edge of the curves than the long wavelength edge. The transmission curve has minimum at the intersection point of two ridge modes; therefore, each edge of the curve arises from a different ridge mode. The mechanism that makes these ridge modes 


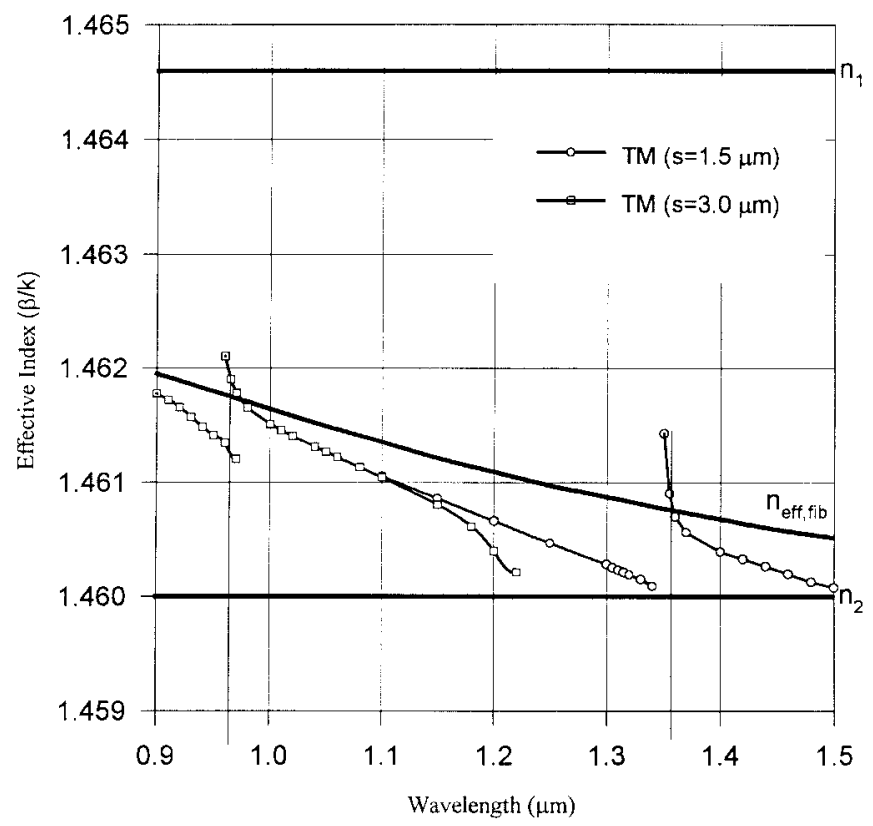

Fig. 7. Effective index as a function of wavelength for TM modes having $s=1.5 \mu \mathrm{m}$ and $s=3.0 \mu \mathrm{m}$.

unbounded at the resonance point is different at two edges of the curves. The longer wavelength edge is formed as the lower order ridge mode slowly diverges from the isolated fiber mode behavior, with more energy transferred into the slab, until its behavior is that of an unbounded slab mode. On the other hand, the short-wavelength edge is formed at the cutoff of the higher order ridge mode. Just below cutoff, the power in this mode is well-confined to the core region of the fiber. Therefore the change in the transmission curve is abrupt. This characteristic can be useful for modulation applications which requires fast dynamic response.

\section{CONCLUSION}

In this paper we have applied a vectorial mode expansion method for the evaluation of the spectral properties of the fiber/slab waveguide system. Our results indicate that the wavelength-filtering properties of the system arise from the degree of boundedness of the ridge mode solution rather than from periodic coupling between slab and fiber modes as predicted by coupled mode theory. The loss mechanism is found to be expansion of the input fiber mode into unbounded slab modes; only when the ridge mode solution approximates the isolated fiber mode is the transmission large. The accuracy of this model is evident in its prediction of the resonant wavelengths and bandwidths of experimentally tested devices [2], [11]

The most important result of this work is the predictive nature of the model for improved device performance. As we have shown, the filter bandwidth may be reduced by increasing the slab index or by increasing the guide separation; the former clearly has a greater effect. We have also shown that the stopband may be tuned by adjusting the superstrate index. This tuning may easily exceed the filter bandwidth, so that good channel isolation may be achieved in WDM applications. In addition, this behavior suggests that improved designs may be developed for such applications as electro-optic modulation, switching, and optical sensing. The accuracy of the model is further supported by its prediction of the existence of the fundamental mode cutoff due to the asymmetry of the structure. Coupled-mode theory predicts no such behavior. Furthermore, coupled mode theory predicts the periodic transfer of power between the fiber and the slab; such behavior has never been observed. Our model accounts for monotonic decrease in power due to loss of boundedness of a single ridge mode.

In conclusion, the model presented here should provide a more accurate analysis tool for design of hybrid fiber/slab filter structures than that afforded by coupled-mode theory, as it eliminates the need for a weak-coupling approximation. The mode expansion technique allows for determination of resonant wavelength, bandwidth, and insertion loss.

\section{REFERENCES}

[1] W. Johnstone, G. Thursby, D. Moodie, R. Varshrey, and B. Culshaw, "Fiber optic wavelength channel selector with high resolution," Electron. Lett., vol. 28, pp. 1364-1366, July 1992.

[2] A. Tz. Andreev, K. P. Panajotov, and E. I. Karakoleva, "Wavelength division action of a distributed single-mode fiber-to-symmetrical planar waveguide coupler," IEEE Photonics Technol. Lett., vol. 6, pp. 1238-1240, Oct. 1994.

[3] G. Raizada and B. P. Pal, "Refractometers and tunable components based on side-polished fibers with multimode overlay waveguides: Role of the superstrate," Opt. Lett., vol. 21, pp. 399-401, Mar. 1996.

[4] S. Zheng, Lee N. Binh, and G. P. Simon, "Light coupling and propagation in composite optical fiber-slab waveguide," J. Lightwave Technol., vol. 13, pp. 244-251, Feb. 1995.

[5] D. Marcuse, "Investigation of coupling between a fiber and infinite slab," J. Lightwave Technol., vol. 7, pp. 122-130, Jan. 1989.

[6] P. P. Krassimir and A. T. Andreev, "Distributed coupling between a single-mode fiber and a planar waveguide," J. Opt. Soc. Amer. B, vol. 11, pp. 826-834, May 1994.

[7] A. W. Snyder and J. D. Love, Optical Waveguide Theory. London, U.K.: Chapman and Hall 1991, ch. 27.

[8] M. S. Dinleyici and D. B. Patterson, "Vector modal solution of evanescent coupler," J. Lightwave Technol., vol. 15, pp. 2316-2324, Dec. 1997.

[9] D. Marcuse, F. Ladouceur, and J. D. Love, "Vector modes of D-shaped fibers," Inst. Elect. Eng. Proc. J., vol. 139, pp. 117-126, Apr. 1992.

[10] C. Vassallo, "Rigorous theory for modes of optical fibers with cladding limited by a plane," Electron. Lett., vol. 22, pp. 944-946, Aug. 1986.

[11] G. Fawcett, W. Johnstone, I. Andonovic, D. J. Bone, T. G. Harvey, N. Carter, and T. G. Ryan, "In-line fiber-optic intensity modulator using electro-optic polymer," Electron. Lett., vol. 28, pp. 985-986, May 1992.

[12] K. McCallion, W. Johnstone, and G. Fawcett, "Tunable in-line fiberoptic bandpass filter," Opt. Lett., vol. 19, pp. 542-544, Apr. 1994.

Mehmet S. Dinleyici received the B.S and M.Sc. degrees in electrical engineering from the University of Gaziantep, Gaziantep, Turkey, in 1991 and 1993, respectively. He received the Ph.D degree in photonics from Illinois Institute of Technology, Chicago.

He is currently with Izmir Institute of Technology, Izmir, Turkey, as an Assistant Professor in the Electrical and Electronics Engineering Department. His interest is in the area of optical fiber devices, electro-optic, dielectric waveguides and photonics devices.

David B. Patterson, photograph and biography not available at the time of publication. 